\title{
A quality improvement project conducted in a GP practice in Greater Manchester to improve the monitoring and safety of patients on apixaban
}

\author{
Author: Zenab Sarwar Mateen
}

\section{Introduction}

Apixaban is a direct oral anticoagulant inhibitor (DOAC) that prevents thrombin generation and thrombus development. ${ }^{1}$ As per National Institute for Health and Care Excellence (NICE) guidelines, those taking apixaban require regular follow up and monitoring due to significant safety issues such as bleeding or renal impairment. ${ }^{1-3}$ In many general practitioner (GP) practices, including Kildonan House, monthly drug safety audits are carried out ${ }^{5}$ and reviewed by the pharmacists. These reports ensure high-risk medications that require regular monitoring are not overlooked as without these requirements prescribers should not be authorising further medication. $^{5}$

However, although rivaroxaban, another DOAC was reviewed, as well as lithium and amiodarone, apixaban was not included. I wanted to put measures in place to improve the system to meet these standards and improve the monitoring and thus safety of patients on apixaban.

\section{Materials and methods}

I conducted the project using Plan, Do, Study, Act quality improvement methodology: a driver diagram was created; outcome and process measures were outlined; a search was conducted on the electronic patient notes; a meeting was set up with the pharmacists to continue implementing the changes made on the electronic system to start monitoring and recalling patients requiring review.

The search was conducted using SystemOne on all patients currently prescribed apixaban $5 \mathrm{mg}$ twice daily and checking:

$>$ if they have a valid indication ${ }^{4}$

$>$ if they are on the correct corresponding dose $\mathrm{e}^{1-3}$

$>$ they have been appropriately reviewed in the last 6 months

$>$ whether their monitoring blood tests in the last 12 months are up to date.

The data was collected and analysed and a results table was created for the following parameters:

$>$ Age of patient

Author: Royal College of Physicians

$>$ Indication

$>$ Weight recorded with date

$>$ Date of bloods (renal function, liver function, full blood count)

$>$ If they fit the criteria for 6 monthly renal function bloods

$>$ Whether any bloods are overdue

$>$ Actions taken from the data collected for each patient

> Whether a review of compliance/side effect was carried out recently.

\section{NICE guidance}

Apixaban licensed for specific indications

Apixaban $5 \mathrm{mg} \mathrm{BD}$ reduced to $2.5 \mathrm{mg} \mathrm{BD}$ if has two of: age $>80$, weight $<61 \mathrm{~kg}$, creatinine $\geq 133$

Apixaban contraindicated if creatinine clearance $(\mathrm{CrCl})$

$<15 \mathrm{~mL} /$ minute.

Apixaban 3 monthly review for compliance, adverse effects and assessment for VTE

Annual routine monitoring (urea and electrolytes (U\&Es), liver function test (LFT), full blood count (FBC))

6-monthly U\&Es if $\mathrm{CrCl}$ $30 ? 60 \mathrm{~mL} / \mathrm{min}$

3-monthly U\&Es if $\mathrm{CrCl}$ $15 ? 30 \mathrm{~mL} / \mathrm{min}$

\section{Results}

3 DVT/PE prophylaxis $=5 \%$ 4 DVT/PE treatment $=7 \%$; 51 patients prophylaxis of stroke $=88 \%$; Therefore $100 \%$ prescribed for licensed indications

$1 / 58=1.7 \%$ One patient flagged up as on wrong dose as met age and weight criteria; ? age >80 (83) ?; weight low (58 kg in 1993)

0 patients had a $\mathrm{CrCl}<15$

In the last 3 months ? 9/58 asked about drug adherence $=16 \%$ ?; $1 / 58$ asked about adverse effects $=1.7 \%$; In the last 6 months ? 23

$19 / 58$ annual bloods overdue $=33 \% ; 11 / 58$ annual bloods were overdue by $<12$ months = $19 \%$; Tasks sent: 11 tasks sent to recall

29/58 needed 6-monthly U\&Es $=50 \% ; 7 / 586$-monthly U\&Es overdue $=12 \%$

$0 / 58$ fit this criteria 


\section{Results and discussion}

$100 \%$ of the patients were prescribed apixaban appropriately and only $1 / 58$ patients was queried as a possible candidate requiring a reduced dose depending on a more recent weight confirming $<61 \mathrm{~kg}$ criterion met. All others were dosed appropriately with the information available.

However, it is evident that we are not meeting standards in the monitoring of apixaban bloods routinely. $33 \%$ of the routine annual bloods were overdue, $19 \%$ of which were $>12$ months overdue. Only $17 \%$ were asked about adverse effects in the last 6 months, and only one of these patients was actually asked in the last 3 months fitting the guidelines. We performed better on documenting drug compliance checked, with $40 \%$ done in the last 6 months but these were all mostly part of complete medication reviews or secondary care reviews rather than apixaban-specific consultations.

I involved practice members to illustrate the importance of adding apixaban to the monthly drug safety report and the following implementations and recommendations were made.

> Add following patient status recall messages:

> all on apixaban need annual U\&Es, LFTs, FBC and $\mathrm{CrCl}$

$>$ all with $\mathrm{CrCl} 30-60$ need 6-monthly U\&Es

$>$ all with $\mathrm{CrCl} 15-30$ need 3-monthly U\&Es

$>$ all with $\mathrm{CrCl}<15$ need apixaban stopped and review

$>$ need updated weight every 5 years for ALL adults.

$>$ Add apixaban on to monthly drug safety report.

> Pharmacists to check during yearly reauthorisation if all routine annual tests completed.

> Pharmacists to review and consider discussing with CCG (clinical commissioning group) about safe prescribing for apixaban and implementing assessment of compliance and adverse effects.

\section{Conclusion}

There is a real risk in drug safety if monitoring requirements are not met as patients are reliant on the prescribing team for authorising their medication as suitable. Although all prescriptions were currently safe, as an ongoing process, the system will now have a fixed report for any patients at risk on apixaban to be identified. Due to the messages flashing on the patient notes they will be more likely to get actioned by staff to change doses and obtain overdue bloods; reducing costs of repeated tests, kinder as less venepuncture, and much safer. The pharmacists were in agreement to monitor those patients highlighted and also as part of their annual reauthorisation review to ensure bloods are up to date. This is therefore a sustainable project and the changes and project have helped to educate the clinicians, pharmacists and patients.

\section{References}

1 National Institute for Health and Care Excellence. Anticoagulation oral. Clinical Knowledge Summary. London: NICE, 2017.

2 National Institute for Health and Care Excellence. Anticoagulants, including non-vitamin K antagonist oral anticoagulants (NOACs). Key therapeutic topic [KTT16]. London: NICE, 2018. Key therapeutic topic (KTT16).

3 Medicines.org.uk. Eliquis $2.5 \mathrm{mg}$ film-coated tablets - Summary of product characteristics (SMPC) - (eMC). EMC, 2019.

4 National Institute for Health and Care Excellence. Atrial fibrillation: management. Clinical guideline [CG180]. London: NICE, 2018.

5 Wood S. A protocol for drugs that require regular monitoring. Prescriber 2014;25:31-5. 